\title{
Content Model Content Type
}

National Cancer Institute

\section{Source}

National Cancer Institute. Content Model Content Type. NCI Thesaurus. Code C115999.

The name of the type of content in a content model. 